\title{
STUDY OF PHENOLOGICAL BEHAVIOR OF PLANTS OF LOWER TANAWAL, ABBOTTABAD, PAKISTAN
}

\author{
Adeela Bibi ${ }^{1} 凹$ \\ ${ }^{1}$ Department of Botany, Hazara University Mansehra-21300, Khyber Pakhtunkhwa, Pakistan.
}

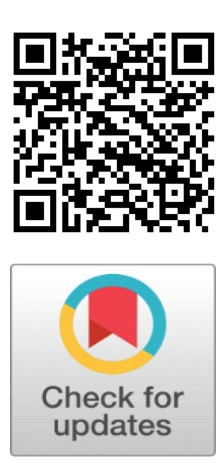

\section{ABSTRACT}

The purpose of this research study to explore the phonological behavior of plants of Lower Tanawal, Pakistan. The phenology of the 286 plants species belonging to 86 families from 80 stands of the Lower Tanawal Pakistan were documented during the different season of the year. It was observed that maximum flowering was recorded in March-April whereas maximum fruiting was noted in June-July. Plant phenology provides knowledge about the effects of environment on flowering and fruiting behavior. This is the first research work on phenology of plants of Lower Tanawal because no work was done on the phenology in past.

Keywords: Phenology, Lower Tanawal, Environment

Received 16 November 2021

Accepted 5 December 2021

Published 31 December 2021

\section{Corresponding Author}

Adeela Bibi, adeelabibi4@gmail.com

DOI

10.29121/granthaalayah.v9.i12.2021 4415

Funding: This research received no specific grant from any funding agency in the public, commercial, or not-for-profit sectors.

Copyright: (C) 2021 The Author(s). This is an open access article distributed under the terms of the Creative Commons Attribution License, which permits unrestricted use, distribution, and reproduction in any medium, provided the original author and source are credited.

\section{INTRODUCTION}

The Lower Tanawal located in District Abbottabad, Province KhyberPakhtunkhwa, Pakistan. The Lower Tanawal located between the Mansehra in the North and the Haripur to the South, and nearby to the Northeast of the Tarbela Lake (Figure 1). The border of Lower Tanawal starts from the village Paswal which is away from Abbottabad on short distance Bibi et al. (2019). The Lower Tanawal is part of the Lesser Himalayas Hussain and Illahi (1991), Gazetteer of the Hazara District (2000).

\section{PLANT PHENOLOGY}

Phenology receives much attention because of global and regional climate change on vegetation. Phenology can also be influenced by geographical factors. The timing of life-cycle of plants events plays an important role in environmental (Zhang et al., 2006).

Phenology is governed by numerous environmental factors, like temperature and moisture Dewald and Steiner (1986). Phenology of plants plays an important role in structured and function of ecosystems Cleland et al. (2007). Changes in plant phenology are due to the early response to global climate change. Because different plant species respond to climate change at different rates Parmesan (2007), Fitter and Fitter (2002), Root et al., 2003). 


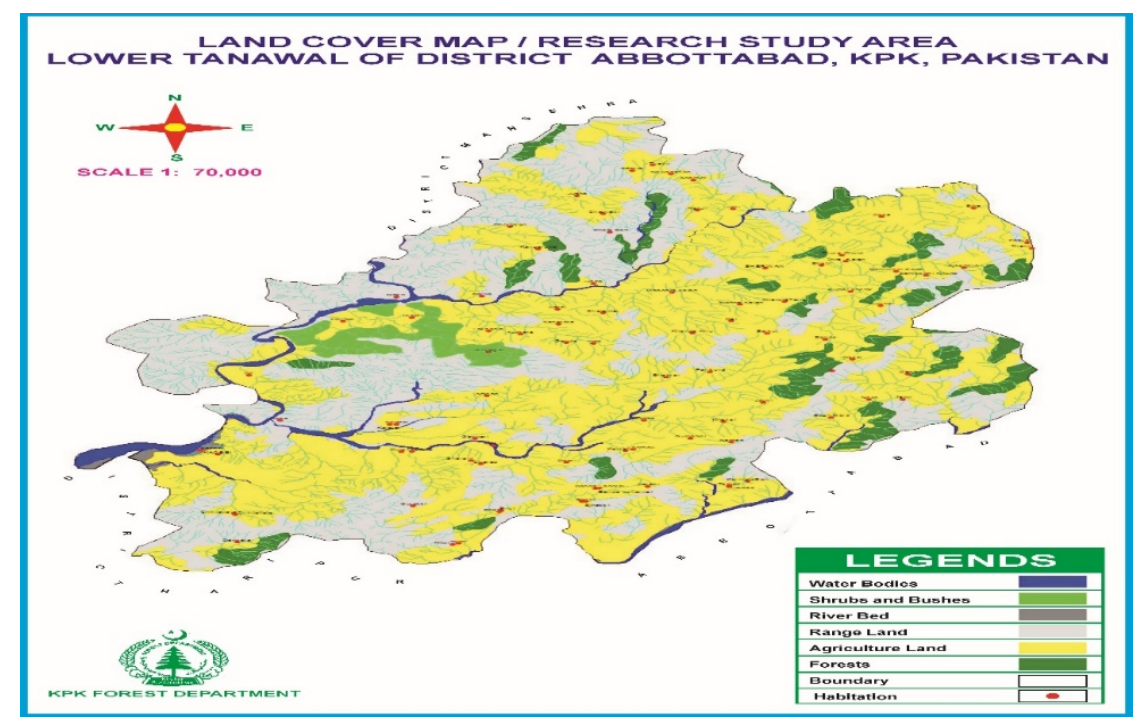

Figure 1 Location Map of Lower Tanawal, Pakistan

The Lower Tanawal, District Abbottabad, Pakistan was chosen for the phonological behavior of plants of Lower Tanawal. This research study was conducted during 20162018. By using Line transect method the 80 localities of Lower Tanawal were selected for this purpose. The study area was frequently visited for collection of data. Data was documented in the field notebook.

\section{RESULTS AND DISCUSSION}

This research study reveals that in past no such study has been conducted on phenology of Lower Tanawal as well as no proper records is present. Thus, our study provides complete information about phonological behavior of plant species of Lower Tanawal. The flowering and fruiting stages of 286 plant species were documented in different seasons. The maximum flowering was recorded in March-April (31.11\%) followed by March-May (26.22\%), while minimum flowering was documented in May-July (0.34\% (Figure 2). The maximum fruiting was noted in June-July (19.93\%) then May-June (19.23\%) while minimum fruiting was noted during August-October $(1.04 \%)$ followed by September-October (0.34\%) (Figure 3). The detail about phenology of plants is given in (Table-1).

\begin{tabular}{rllll}
\hline S. No. & \multicolumn{1}{c}{ Plant Name } & Family & Flowering & Fruiting \\
\hline 1. & Acacia mearnsii De wild. & Fabaceae & May-June & July-Aug \\
\hline 2. & Acacia modesta Wall. & Fabaceae & May-June & Apr-July \\
\hline 3. & Acacia nilotica (L.) & Fabaceae & Apr-May & June-July \\
\hline 4. & Acer pseudoplatanus L. & Sapindaceae & Mar-Apr & Apr-July \\
\hline 5. & Achyranthes aspera. L & Amaranthaceae & Mar-Apr & July-Aug \\
6. & Adiantum capillus-veneris L. & Pteridaceae & May-June & Sep-Oct \\
7. & Aerva javanica (Burn.f.) Juss.ex Shult. & Amaranthaceae & Mar-Apr & June-July
\end{tabular}


Adeela Bibi

\begin{tabular}{|c|c|c|c|c|}
\hline 8. & $\begin{array}{l}\text { Aesculus indica (Wall.ex cambss.) } \\
\text { Hook. }\end{array}$ & Sapindaceae & May-June & June-Sep \\
\hline 9. & Ajuga bracteosa Wall. ex Benth. & Lamiaceae & Mar-May & June-Aug \\
\hline 10. & Albizia lebbeck (L.) Benth. & Fabaceae & Mar-May & May-Sep \\
\hline 11. & Allium griffithianum Boiss. & Amaryllidaceae & Mar-May & June-July \\
\hline 12. & Alliaria petiolata (M. Bieb.) Cavara & Brassicaceae & Mar-Apr & June-Aug \\
\hline 13. & Allium stipitatum Regel & Amaryllidaceae & Apr-May & June-July \\
\hline 14. & Alnus nitida (Spach.) Endl. & Betulaceae & June-July & Aug-Oct \\
\hline 15. & Alternanthera pungens Kunth. & Amaranthaceae & Apr-May & July-Aug \\
\hline 16. & Amaranthus viridis $\mathrm{L}$. & Amaranthaceae & Mar-Apr & June-July \\
\hline 17. & Anagallis arvensis $\mathrm{L}$. & Primulaceae & Mar-Apr & June-July \\
\hline 18. & $\begin{array}{l}\text { Anaphalis margaritacea (L.) Benth. } \\
\text { Hook.f. }\end{array}$ & Asteraceae & Apr-June & July-Sep \\
\hline 19. & $\begin{array}{l}\text { Anaphalis triplinervis (Sims) Sims ex } \\
\text { C.B. }\end{array}$ & Asteraceae & Apr-May & July-Aug \\
\hline 20. & Angelica arguta Nutt. & Apiaceae & Mar-June & June-Aug \\
\hline 21. & $\begin{array}{l}\text { Andrachne cordifolia (Decne.) } \\
\text { Müll.Arg }\end{array}$ & Euphorbiaceae & Apr-June & June-July \\
\hline 22. & Androsace rotundifolia Hardw. & Primulaceae & Mar-Apr & May-June \\
\hline 23. & Anthriscus caucalis M. Bieb. & Apiaceae & Apr-June & June-July \\
\hline 24. & Argemone Mexicana L. & Papaveraceae & May-July & Aug-Oct \\
\hline 25. & Arisaema flavum (Forssk.) Schott & Araceae & Apr-June & July-Aug \\
\hline 26. & Aristida cyanantha Steud. & Poaceae & Apr-June & June-July \\
\hline 27. & Artemisia absinthium L. & Asteraceae & Mar-Apr & May-June \\
\hline 28. & Artemisia scoparia Waldst. \& Kitam. & Asteraceae & Apr-May & June-July \\
\hline 29. & Arundo donax L. & Poaceae & June- July & July-Sep \\
\hline 30. & Asparagus adscendens Roxb. & Asparagaceae & Apr-May & May-Aug \\
\hline 31. & Asparagus officinalis L. & Asparagaceae & Mar-June & June-Aug \\
\hline 32. & Asphodelus tenuifolius Cav. & Asphodelaceae & Apr-July & July-Sep \\
\hline 33. & Asplenium ceterach. L & Aspleniaceae & June-July & July-Aug \\
\hline 34. & Asplenium trichomanes L. & Aspleniaceae & June-July & July-Aug \\
\hline 35. & $\begin{array}{l}\text { Astragalus leucocephalus Grah. ex } \\
\text { Benth. }\end{array}$ & Fabaceae & Mar-May & June-July \\
\hline 36. & Barleria cristata L. & Acanthaceae & Mar-June & June-Sep \\
\hline 37. & Bauhinia variegata L. & Fabaceae & Mar-Apr & May-June \\
\hline 38. & Berberis lycium Royle & Berberidaceae & Mar-June & June-July \\
\hline
\end{tabular}


Study of Phenological Behavior of Plants of Lower Tanawal, Abbottabad, Pakistan

\begin{tabular}{|c|c|c|c|c|}
\hline 39. & Biden pilosa $\mathrm{L}$. & Asteraceae & Mar-June & July-Aug \\
\hline 40. & Boerhavia diffusa L. & Nyctaginaceae & Mar-Apr & Apr- May \\
\hline 41. & Bothriochloa ischaemum (L). Keng. & Poaceae & Apr-May & June-July \\
\hline 42. & Bromus tectorum L. & Poaceae & Mar-Apr & Apr-July \\
\hline 43. & Broussonetia papyrifera L. & Moraceae & Apr-May & May-July \\
\hline 44. & Buddleja asiatica Lour. & Buddlejaceae & Mar-May & May-June \\
\hline 45. & Caesalpinia pulcherrima (L.) Sw. & Fabaceae & Mar-Apr & Apr-June \\
\hline 46. & $\begin{array}{l}\text { Calamintha hydaspidis (Falconer ex } \\
\text { Benth.) }\end{array}$ & Lamiaceae & Mar-Apr & Apr-June \\
\hline 47. & Calamintha nepeta L. & Lamiaceae & Mar-Apr & May-June \\
\hline 48. & $\begin{array}{l}\text { Calamagrostis acutiflora (Schrad.) } \\
\text { DC. }\end{array}$ & Poaceae & Mar-May & June-July \\
\hline 49. & Campanula leucoclada Boiss. & Campanulaceae & Apr-June & June-July \\
\hline 50. & Cannabis sativa L. & Cannabaceae & Mar-June & Mar-June \\
\hline 51. & Canna indica $\mathrm{L}$. & Cannabaceae & Mar-Apr & Apr-July \\
\hline 52. & Capsella bursa Raf. & Brassicaceae & Mar-May & June-Aug \\
\hline 53. & Cardamine hirsuta L. & Brassicaceae & Mar-Apr & May-June \\
\hline 54. & Carduus nutans L. & Asteraceae & Apr-May & June-Sep \\
\hline 55. & Carissa opaca Stapf ex Haines & Apocynaceae & Apr-June & June-July \\
\hline 56. & Caryopteris odorata (Ham. ex Roxb.). & Verbenaceae & Mar-Apr & Apr-June \\
\hline 57. & Carthamus oxyacantha M. Bieb. & Asteraceae & Mar-Apr & May-June \\
\hline 58. & Cedrela serrata Royle & Meliaceae & Apr-May & June-July \\
\hline 59. & Celtis australis $\mathrm{L}$. & Cannabaceae & Mar-Apr & Apr-June \\
\hline 60. & Chenopodium album L. & Chenopodiaceae & Mar-Apr & Apr-May \\
\hline 61. & Chenopodium ambrosioides L. & Chenopodiaceae & Mar-May & June-July \\
\hline 62. & Chenopodium vulgare $\mathrm{L}$. & Chenopodiaceae & Mar-Apr & May-June \\
\hline 63. & Chrysopogon serrulatus Trin. & Poaceae & Mar-May & June-July \\
\hline 64. & Cichorium intybus L. & Asteraceae & Mar-Apr & May-June \\
\hline 65. & Cirsium falconeri (Hook.f.) Petr. & Asteraceae & Mar-May & May-June \\
\hline 66. & Cissampelos pareira L. & Menispermaceae & Apr-June & June-July \\
\hline 67. & Citrullus colocynthis (L.) Schrad. & Cucurbitaceae & Apr-June & June-Sep \\
\hline 68. & Clematis grata Wall. & Ranunculaceae & June-July & July-Sep \\
\hline 69. & Clematis graveolens Lindl. & Ranunculaceae & June-July & July-Sep \\
\hline 70. & Colebrookea oppositifolia Sm. & Lamiaceae & Apr-June & June-July \\
\hline 71. & Commelina virginica L. & Commelinaceae & Apr-June & June-Sep \\
\hline
\end{tabular}


Adeela Bibi

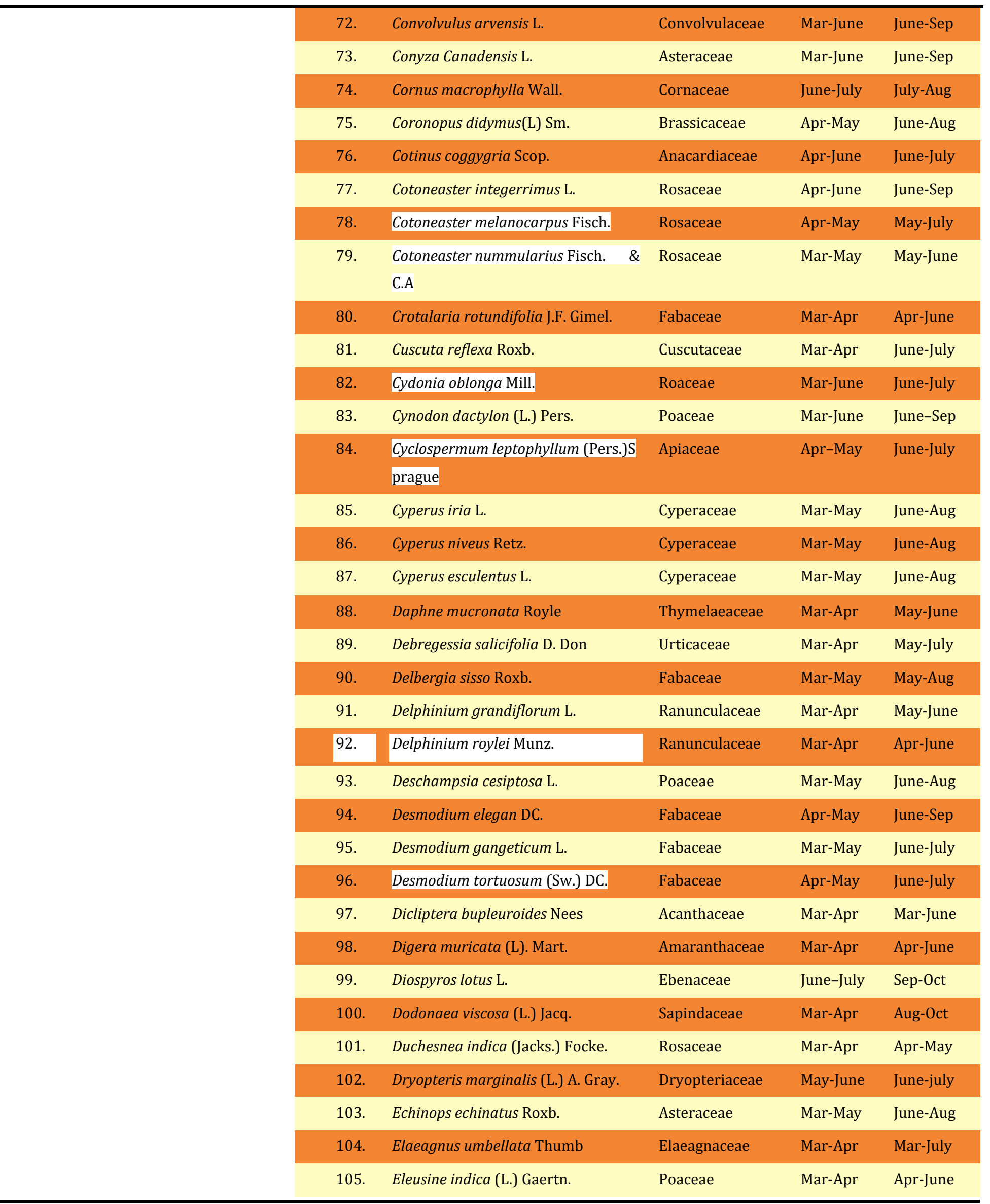


Study of Phenological Behavior of Plants of Lower Tanawal, Abbottabad, Pakistan

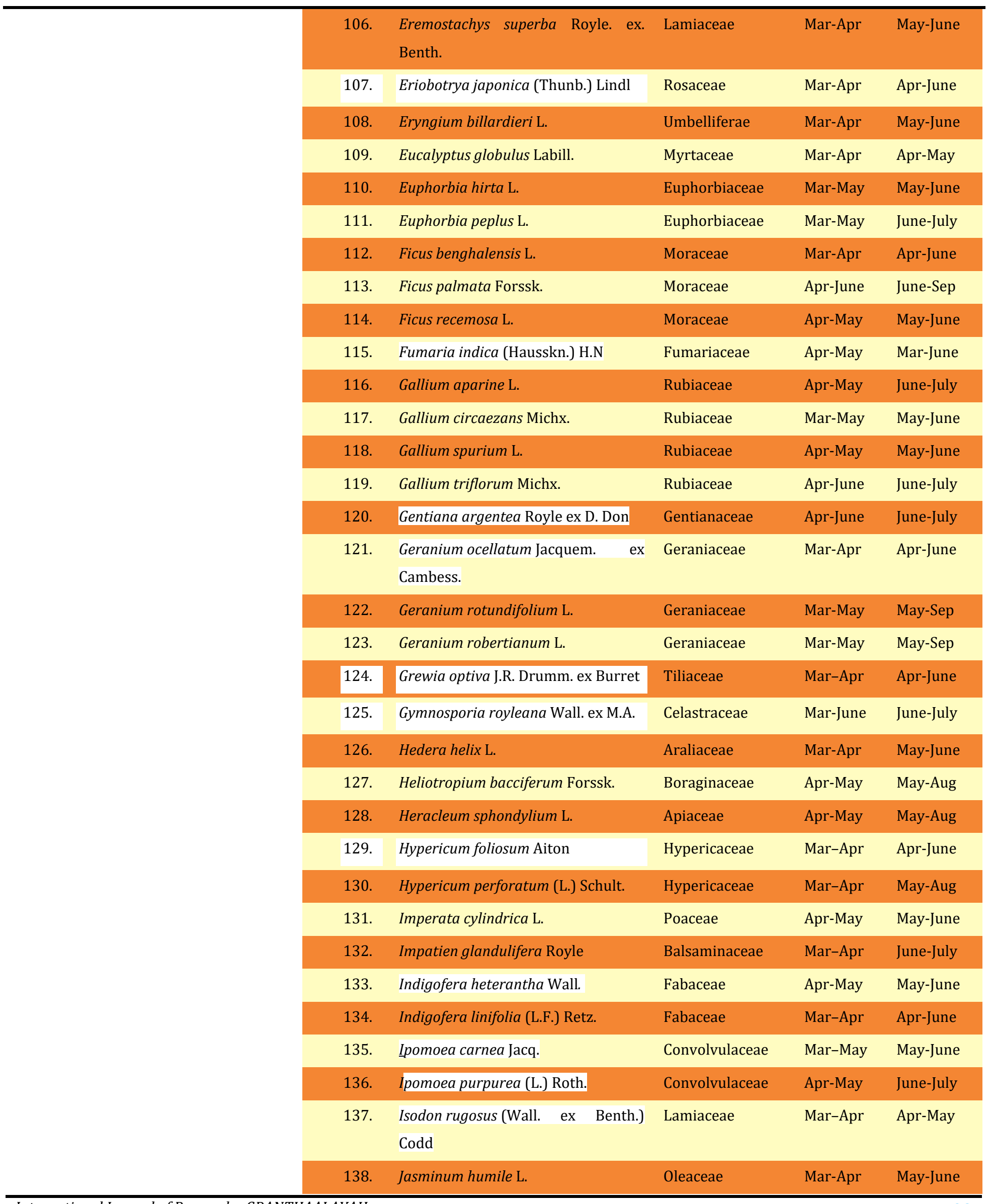




\begin{tabular}{|c|c|c|c|c|}
\hline 139. & Juglan regia $\mathrm{L}$. & Juglandaceae & May-June & June-July \\
\hline 140. & Justicia adhatoda $\mathrm{L}$. & Acanthaceae & Mar-Apr & Apr-May \\
\hline 141. & Justicia pectoralis Jacq. & Acanthaceae & Mar-Apr & May-June \\
\hline 142. & Kickxia ramosissima Wall. & Scrophulariaceae & Mar-May & May-June \\
\hline 143. & Lactuca indica $\mathrm{L}$. & Asteraceae & Mar-Apr & Apr-May \\
\hline 144. & Lactuca serriola L. & Asteeaceae & May-June & June-July \\
\hline 145. & Lamium album $\mathrm{L}$. & Lamiaceae & Mar-May & May-June \\
\hline 146. & Lannea coromandelica (Houtt). Merr & Anacardiaceae & Apr-June & July-Aug \\
\hline 147. & Lantana camara L. & Verbenaceae & Mar-Apr & Apr-June \\
\hline 148. & Lappula barbata (M. Bieb.) Gürke & Boraginaceae & Apr-May & May-June \\
\hline 149. & Lathyrus aphaca L. & Fabaceae & Apr-May & May-June \\
\hline 150. & $\begin{array}{l}\text { Launaea procumbens (Roxb.) } \\
\text { Ramayya \& Rajagopal }\end{array}$ & Asteraceae & Mar-June & June-July \\
\hline 151. & Lepidium didymium $\mathrm{L}$. & Brassicaceae & Mar-Apr & Apr-July \\
\hline 152. & $\begin{array}{l}\text { Leptodermis virgata Edgew. } \\
\text { Hook.f. }\end{array}$ & Rubiaceae & Mar-Apr & May-June \\
\hline 153. & Lespedeza juncea Linn.f & Fabaceae & Mar-June & June-July \\
\hline 154. & Linum corymbulosum Rchb. & Linaceae & Mar-Apr & Apr-June \\
\hline 155. & Limonium echioides (L.) Mill. & Plumbaginaceae & Mar-May & May-Sep \\
\hline 156. & Lonicera quinquelocularis Hard. & Carprifoliaceae & Mar-June & June-July \\
\hline 157. & $\begin{array}{l}\text { Malvastrum coromandelianum (L.) } \\
\text { Garcke }\end{array}$ & Malvaceae & Mar-May & May-Sep \\
\hline 158. & $\begin{array}{l}\text { Mallotus philippensis (Lam) } \\
\text { Muell.Arg. }\end{array}$ & Euphorbiaceae & Feb-Apr & Apr-May \\
\hline 159. & Malva neglecta Wallr. & Malvaceae & Mar-June & June-July \\
\hline 160. & Marrubium vulgare L. & Lamiaceae & Mar-May & June-July \\
\hline 161. & Melia azedarach L. & Meliaceae & Mar-Apr & Apr-July \\
\hline 162. & Medicago denticulata Willd. & Fabaceae & Apr-May & May-June \\
\hline 163. & Medicago polymorpha L. & Fabaceae & Mar-June & June-July \\
\hline 164. & Melilotus indicus (L). All. & Fabaceae & Mar-May & May-July \\
\hline 165. & Mentha arvensis $\mathrm{L}$ & Lamiaceae & Mar-June & June-Sep \\
\hline 166. & Mentha longifolia (L.) Huds & Lamiaceae & Mar-Apr & Apr-July \\
\hline 167. & $\begin{array}{l}\text { Micromeria biflora (Buch. D. Don) } \\
\text { Benth. }\end{array}$ & Lamiaceae & Apr-May & May-June \\
\hline 168. & Mirabilis jalapa L. & Nyctaginaceae & Apr-May & May-June \\
\hline 169. & Morus alba L. & Moraceae & June-July & Aug-Sep \\
\hline
\end{tabular}


Study of Phenological Behavior of Plants of Lower Tanawal, Abbottabad, Pakistan

\begin{tabular}{|c|c|c|c|c|}
\hline 170. & Morus nigra L. & Moraceae & June-July & Aug-Sep \\
\hline 171. & Myrsine africana $\mathrm{L}$. & Primulaceae & Mar-Apr & May-June \\
\hline 172. & Nasturtium officinale R.Br. & Brassicaceae & Mar-Apr & Apr-July \\
\hline 173. & $\begin{array}{l}\text { Nanorrhinum acerbianum (Boiss.) } \\
\text { Betsche }\end{array}$ & Plantaginaceae & Apr-July & Aug-Sep \\
\hline 174. & $\begin{array}{l}\text { Nannorrhop ritchiana (Griff). } \\
\text { Aitchison. }\end{array}$ & Arecaceae & Apr-July & Aug-Sep \\
\hline 175. & Nepeta cataria L. & Lamiaceae & Mar-Apr & Apr-May \\
\hline 176. & Nepeta ciliaris Benth. & Lamiaceae & Mar-Apr & May-July \\
\hline 177. & $\begin{array}{l}\text { Notholirion thomsonianum (Royle) } \\
\text { Stapf }\end{array}$ & Liliaceae & Mar-Apr & Apr-May \\
\hline 178. & Oenothera rosea L. Her. ex Aiton & Onagraceae & Mar-Apr & Apr-July \\
\hline 179. & Olea ferruginea Royle. & Oleaceae & Apr-May & May-June \\
\hline 180. & Onosma hispida Wall. ex G. Don & Boraginaceae & Mar-May & May-July \\
\hline 181. & Opuntia ovata Pfeiff. & Cactaceae & Mar-Apr & Apr-July \\
\hline 182. & Origanum vulgare L. & Lamiaceae & Apr-May & June-Sep \\
\hline 183. & Otostegia limbata (Benth.) Boiss. & Lamiaceae & Mar-Apr & May-July \\
\hline 184. & Oxalis corniculata $\mathrm{L}$. & Oxalidaceae & Mar-Apr & May-June \\
\hline 185. & Parthenium hysterophorus L. & Asteraceae & Mar-Apr & May-June \\
\hline 186. & Pennisetum orientale. Rich. & Poaceae & Mar-May & June-July \\
\hline 187. & Pentanema vestitum Wall.ex. DC & Asteraceae & Mar-Apr & May-June \\
\hline 188. & Periploca aphylla. Decne. & Apocynaceae & Mar-May & May-July \\
\hline 189. & Perilla frutescens (L) Britton. & Lamiaceae & Mar-May & June-July \\
\hline 190. & Phleum alpinum L. & Poaceae & Mar-June & June-July \\
\hline 191. & Phleum arenarium $\mathrm{L}$. & Poaceae & Mar-Apr & May-July \\
\hline 192. & Pimpinella stewartii Nasir & Apiaceae & Mar-Apr & Apr-July \\
\hline 193. & Pinus roxburghii Sarg. & Pinaceae & Mar-Apr & Apr-July \\
\hline 194. & $\begin{array}{l}\text { Pistacia integerrima J.L. Stewart. ex. } \\
\text { Brands. }\end{array}$ & Anacardiaceae & Mar-May & May-July \\
\hline 195. & Plantago lanceolata $\mathrm{L}$. & Plantaginaceae & Mar-May & May-June \\
\hline 196. & Plumbago zeylanica L. & Plumbaginaceae & Mar-Apr & Apr-July \\
\hline 197. & Poa balfourii Auct. & Poaceae & Apr-May & June-July \\
\hline 198. & Poa poiformis Labill & Poaceae & Mar-May & May-July \\
\hline 199. & Polygala abyssinica R.Br.ex. Fresen. & Polygalaceae & Apr-May & May-June \\
\hline 200. & Polygala vulgaris L. & Polygonaceae & Mar-Apr & Apr-July \\
\hline 201. & Polygonum plebeium R.Br. & Polygonaceae & Mar-May & May-July \\
\hline
\end{tabular}


Adeela Bibi

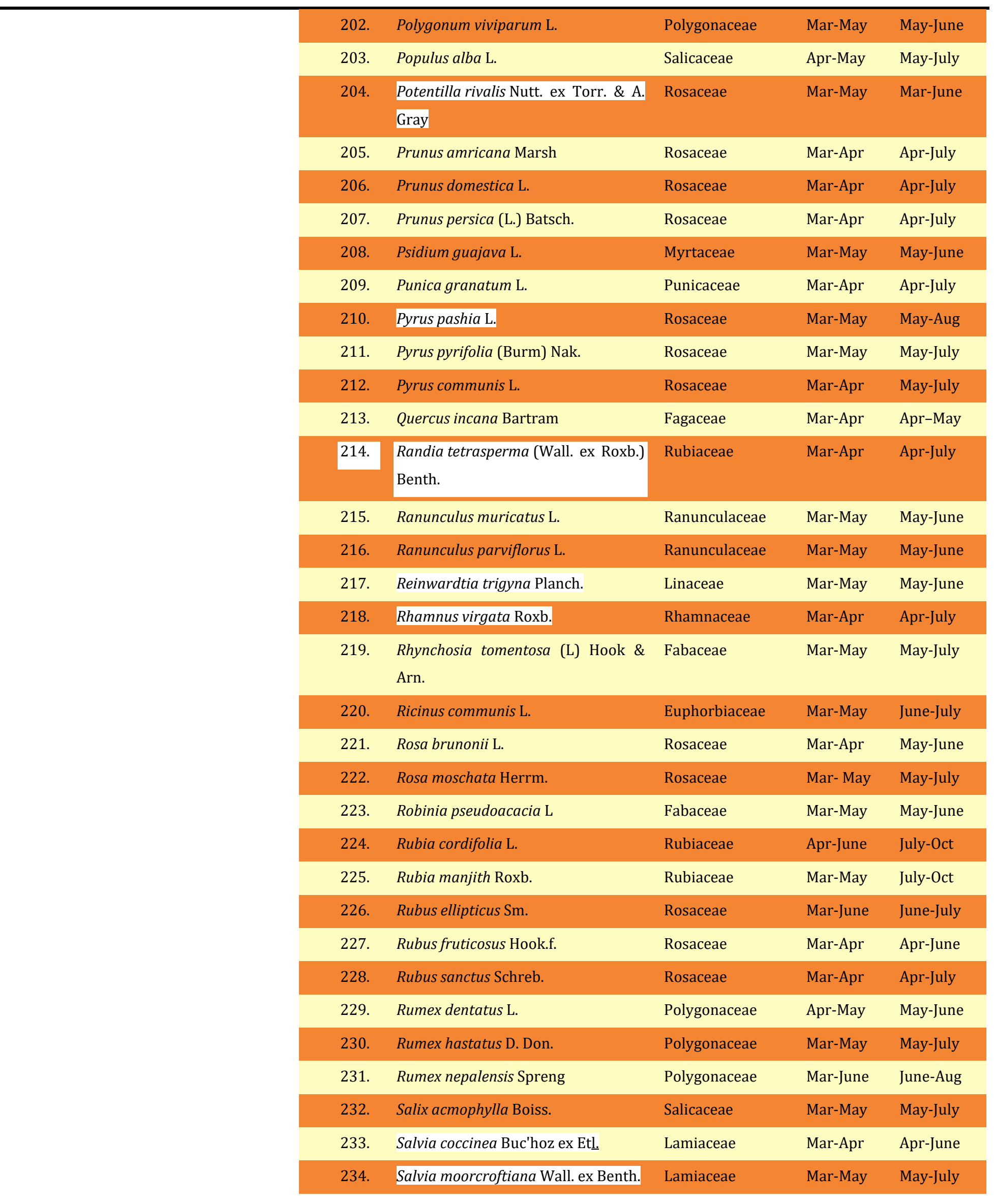


Study of Phenological Behavior of Plants of Lower Tanawal, Abbottabad, Pakistan

\begin{tabular}{|c|c|c|c|c|}
\hline 235. & Sapindus mukorossi Gaertn & Sapindaceae & Mar-Apr & Apr-Aug \\
\hline 236. & $\begin{array}{l}\text { Saussurea heteromalla (D. Don) } \\
\text { Hand- }\end{array}$ & Asteraceae & Mar-May & May-July \\
\hline 237. & Scabiosa candollei Wall. & Dipsaceae & Mar-Apr & Apr-May \\
\hline 238. & Scabiosa ochroleuca L. & Caprifoliaceae & Mar-May & May-July \\
\hline 239. & Scandix pectin -veneris L. & Apiaceae & Mar-May & May-Aug \\
\hline 240. & Scilla griffithii Hochr. & Hyacinthaceae & Mar-Apr & May-July \\
\hline 241. & $\begin{array}{l}\text { Scrophularia dentata Royle. ex. } \\
\text { Bentham }\end{array}$ & Scrophulariaceae & Mar-May & May-Sep \\
\hline 242. & Sedum sarmentosum Bunge & Crassulaceae & Mar-Apr & Apr-June \\
\hline 243. & Senecio aquaticus Hill. & Asteraceae & Mar-May & June-July \\
\hline 244. & Sida cordata (Burm.f.) Borss.Waalk & Malvaceae & Mar-May & May-July \\
\hline 245. & Sisymbrium irio L. & Brassicaceae & Feb-Apr & Apr-May \\
\hline 246. & Silene conoidea L. & Caryophyllaceae & Mar-May & May-June \\
\hline 247. & Smilax -bona-nox L. & Smilacaceae & Mar-May & May-Sep \\
\hline 248. & Smilax china L. & Smilacaceae & Apr-May & May-Sep \\
\hline 249. & Smilax rotundifolia $\mathrm{L}$. & Smilacaceae & Mar-May & May-Sep \\
\hline 250. & Solanum erianthum L. & Solanaceae & Apr-May & May-June \\
\hline 251. & Solanum incanum L. & Solanaceae & Mar-May & May-July \\
\hline 252. & Solanum nigrum L. & Solanaceae & Mar-May & May-June \\
\hline 253. & Solanum surattense L. & Solanaceae & Apr-May & May-July \\
\hline 254. & Sonchus asper (L.) Hill & Asteraceae & Apr-May & June-July \\
\hline 255. & Sonchus oleraceus (L.) Hill & Asteraceae & Mar-Apr & Apr-July \\
\hline 256. & Sorbaria tomentosa (Lindl.) Rehder & Rosaceae & Mar-May & June-Aug \\
\hline 257. & Stachys emodi. Hedge. & Lamiaceae & Apr-May & May-June \\
\hline 258. & Spiraea vaccinifolia D. Don & Rosaceae & Mar-Apr & May-July \\
\hline 259. & Stellaria alsinoides Boiss. & Caryophyllaceae & Mar-May & May-June \\
\hline 260. & Stellaria media (L.) Vill. & Caryophyllaceae & Mar-May & May-Sep \\
\hline 261. & $\begin{array}{l}\text { Strobilanthes efloraofindia C.B. } \\
\text { Clarke }\end{array}$ & Acanthaceae & Mar-May & May-Aug \\
\hline 262. & Strobilanthes oliganthus Miq. & Acanthaceae & Mar-Apr & Apr-Aug \\
\hline 263. & Symplocos recemosa Roxb. & Symplocaceae & Mar-May & May-July \\
\hline 264. & Tagetes minuta L. & Asteraceae & Apr-May & June-July \\
\hline 265. & Taraxacum officinale $\mathrm{L}$. & Asteraceae & Mar-Apr & Apr-May \\
\hline 266. & Tricholepis angustifolia-DC & Asteraceae & Mar-Apr & Apr-Aug \\
\hline 267. & Trichodesma indicum(L)R. Br. & Boraginaceae & Mar-May & May-July \\
\hline
\end{tabular}




\begin{tabular}{|c|c|c|c|c|}
\hline 268. & Tridax procumbens L. & Asteraceae & Mar-May & May-June \\
\hline 269. & Trifolium repens $\mathrm{L}$. & Fabaceae & Mar-Apr & May-Aug \\
\hline 270. & Ulmus villosa Brandes.ex. Gamble & Ulmaceae & Mar-May & May-Sep \\
\hline 271. & Verbena officinalis L. & Verbenaceae & Apr-May & June-July \\
\hline 272. & Veronica stewartii Pennel & Plantaginaceae & Apr-July & July-Sep \\
\hline 273. & Verbena tenuisecta Briq. & Verbenaceae & Mar-May & Mar-May \\
\hline 274. & Verbascum Thapsus L. & Scrophulariaceae & May-June & June-Sep \\
\hline 275. & Viburnum cotinifolium L. & Caprifoliaceae & Apr-May & May-July \\
\hline 276. & Vicia hirsuta (L.) Gray. & Fabaceae & Mar-June & July-Sep \\
\hline 277. & Vicia sativa $\mathrm{L}$. & Fabaceae & Mar-May & Mar-June \\
\hline 278. & Viola Odorata L. & Violaceae & Mar-Apr & Apr-May \\
\hline 279. & Vitex negundo L. & Verbenaceae & Apr-May & May-Aug \\
\hline 280. & Vitis vinifera $\mathrm{L}$. & Vitaceae & Mar-Apr & Apr-July \\
\hline 281. & Woodfordia fruticosa (L.) Kurz. & Lythraceae & Feb-Apr & Apr-June \\
\hline 282. & Xanthium strumarium L. & Asteraceae & Mar-May & Mar-June \\
\hline 283. & Zanthoxylum armatum Dc. & Rutaceae & Apr-May & May-June \\
\hline 284. & Ziziphus oenopila L. & Rhamnaceae & Feb-Apr & Apr-June \\
\hline 285. & Ziziphus nummularia (Burm.f.) & Rhamnaceae & Mar-May & Mar-June \\
\hline & Wight & & & \\
\hline 286. & Ziziphus jujuba Mill. & Rhamnaceae & Mar-Apr & May-June \\
\hline
\end{tabular}

These results are in line with the Kikim and Yadava (2001) they stated that in the Sub-tropical forests of Manipur India mostly flowers occur from April to September. The change in climatic conditions can affect the flowering period of plant species. These results are also in accordance with Khan et al. (2016) who indicated that maximum flowering was noted in the month of March-April in Sathan Galli Hill, Pakistan. Our findings are also agreed with Amjad et al. (2013) who described the phenological behavior of plants from Nikyal Valley, Pakistan and reported that maximum fruiting was observed in June.

This study would be helpful in knowing the timing of different phenological phases of the plants which can be of interest to people of the area who wish to plan their gardens round the year. This study would also be helpful for making comparison over long time. For example, if there is any change in the phenological behavior of the same plant species in next 5 years. Then such study could not be possible at this time as no relative literature is available for this area.

The period of maximum flowering activity in the months of spring agreed with the observations of Pilar and Gabriel. Flower development periods of the same plant species seems to be important in increasing the chances of pollinations as proposed by Ollerton and Lack.The changes in phonological behavior of plants are due to the temperature and rainfall Parmesan (2007). The study provides information about change in climatic conditions which affect the flowering period of plant species. 
People of the study area are usually unaware of the importance of phenology in their daily lives. The present understanding of phenology is significant to help people know how plant species respond to climate change.

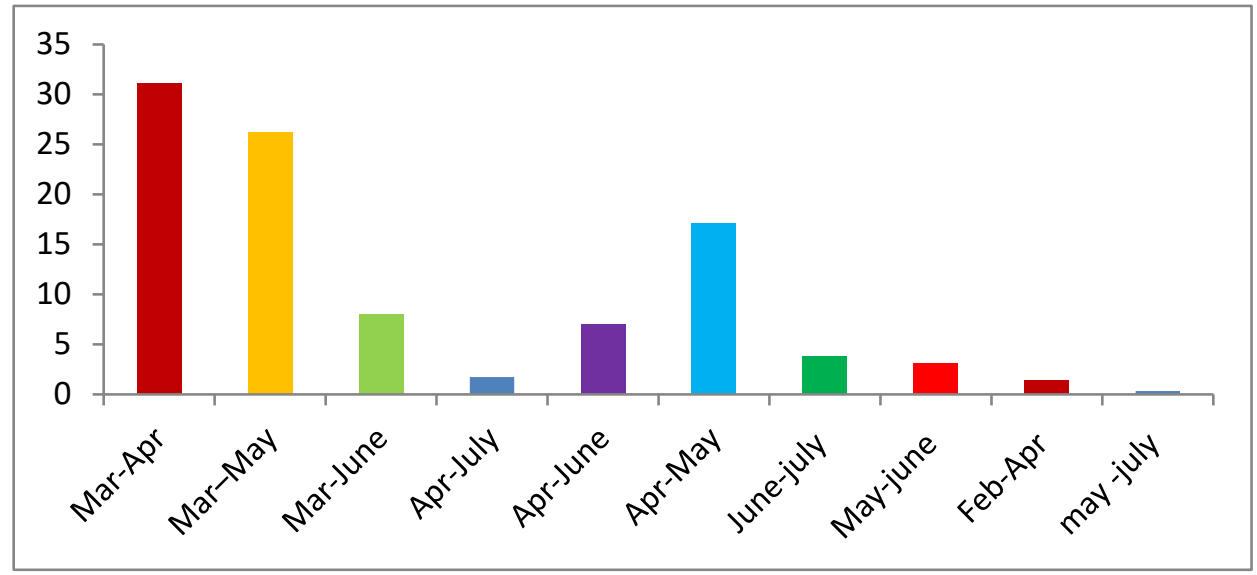

Figure 2 Graph showing flowering period of plant species

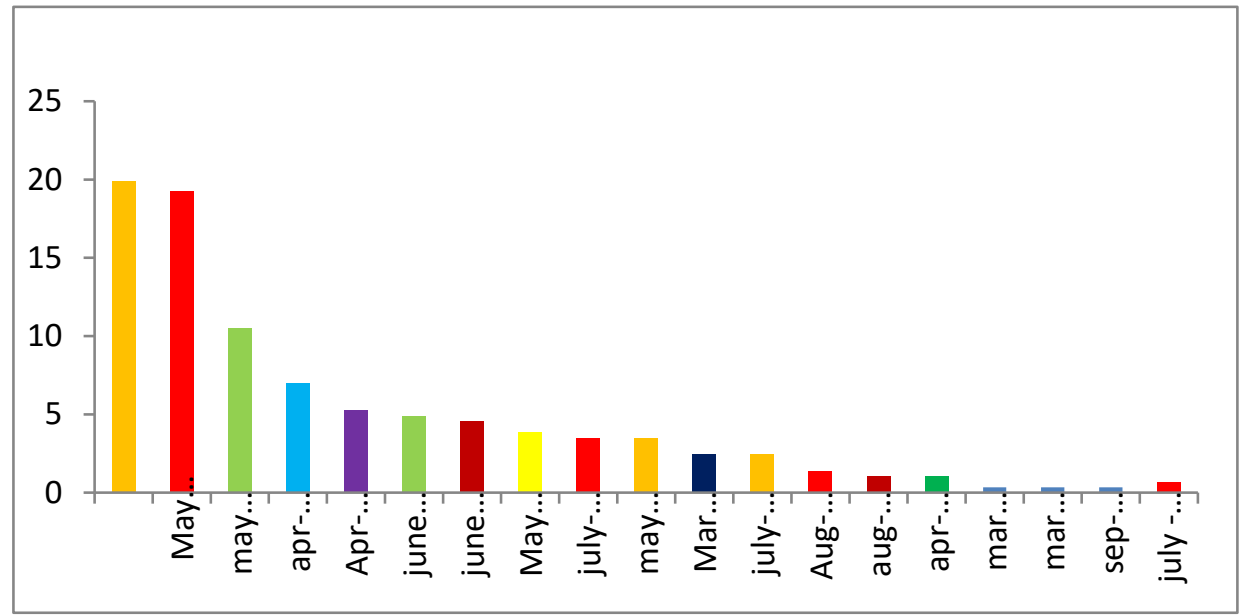

Figure 3 Graph showing fruiting period of plant species

\section{REFERENCES}

Amjad, M. S., M. Arshad and S. K. Chaudhari. (2013). Phenological patterns among the vegetation of Nikyal valley, District Kotli, Azad Jammu and Kashmir, Pakistan. British J. Applied Sci. \& Tech., 3(4): 1505-1518. Retrieved from https://doi.org/10.9734/BJAST/2014/5329

Bibi, A. Z. Iqbal, G.M. Shah. (2019). Floristic diversity, biological spectrum of Lower Tanawal, KP, Pakistan. Ukr. J. Ecol., 4 (9): 505-514. Retrieved from https://doi.org/10.15421/2019_782

C.-D. Pilar and M.-M. Gabriel, (1998) "Phenological pattern of fifteen Mediterranean phanaerophytes from Quercus ilex communities of NE-Spain," Plant Ecology, vol. 139, no. 1, pp. 103-112. Retrieved from https://doi.org/10.1023/A:1009759318927 
Cleland, E.E., Chuine, I., Menzel, A., Mooney, H.A. and Schwartz, M.D. (2007). Shifting plant phenology in response to global change. Trends Ecol. Evol., 22 : 357365. Retrieved from https://doi.org/10.1016/j.tree.2007.04.003

Dewald, L.E. and Steiner, K.C. (1986). Phenology, height increment and cold tolerance of Almus glutinosa population in a common environment. Silvae Genetica, 35 : 205-211. Retrieved from http://www.sauerlaenderverlag.com/CMS/fileadmin/content/dokument/archiv/silvaegenetica/35_ 1986/35-5-6-205.pdf

Fitter, A.H., and Fitter, S.R. (2002). Rapid change in flowering time in British plants. Science, 296 : 1689-1691. Retrieved from https://doi.org/10.1126/science.1071617

Gazetteer of the Hazara District (1883) -4. Sang-e-Meel Publications, 2000-Hazara District (Pakistan).

Hussain, F., and I. Illahi. (1991). Ecology and Vegetation of Lesser Himalayan Pakistan. Botany Department University of Peshawar, pp. 187.

J. Ollerton and A. J. Lack, (1992) "Flowering phenology: an example of relaxation of natural selection?" Trends in Ecology and Evolution, vol. 7, no. 8, pp. 274276. Retrieved from https://doi.org/10.1016/0169-5347(92)90175-B

Kikim, A. and P. S. Yadava. (2001). Phenology of tree species in subtropical forests of Manipur in north eastern India. Trop. Ecol., 42(2): 269-276. Retrieved from https://tropecol.com/pdf/open/PDF_42_2/42212.pdf

Parmesan, C. (2007). Influence of species, latitudes, and methodologies on estimates of phenological response to global warming. Global Change Biology. 13 : 1860-1872, 2007. Retrieved from https://doi.org/10.1111/j.13652486.2007.01404.x

Watson, H.D. (1907) Gazetteer of the Hazara District, Sarhad Urdu Academy NWFP, Pakisan 